\title{
Desarrollo productivo de dos variedades locales de maíz (Zea mays $L$.) con la aplicación de fertilizante mineral y orgánico en la Amazonía Ecuatoriana
}

\section{Productive development of two local varieties of maize (Zea mays L.) with the application of mineral and organic fertilizer in the Ecuadorian Amazon}

Reinaldo Demesio Alemán Pérez ${ }^{1 *}$, Ronnal Vinicio Ortiz Tenemaza ${ }^{2}$, Javier Domínguez Brito ${ }^{1}$, Carlos Alfredo Bravo Medina ${ }^{1}$, Jorge Luis Alba Roja ${ }^{1}$, Yoel Rodríguez Guerra ${ }^{1}$, Carlos Pico Angulo ${ }^{1}$, Jorge Freile Almeida ${ }^{1}$

${ }^{1}$ Departamento de Ciencias de la tierra, Universidad Estatal Amazónica, Puyo, Ecuador ${ }^{2}$ Graduado de Maestría en Agronomía, Universidad Estatal Amazónica, Puyo, Ecuador

*Correspondencia: raleman@uea.edu.ec

\section{Resumen}

$\mathrm{E}^{\mathrm{l}}$ maíz es uno de los principales granos en el Ecuador, de gran importancia para los pueblos indígenas, quienes lo consideran un generador de vida. En la región Amazónica se viene cultivando desde hace muchos años las variedades Tusilla y Zhubay, sin embargo, no existen estudios agronómicos que permitan determinar un modelo de producción para la región. En base a ello, el objetivo de este trabajo consistió en evaluar el desarrollo productivo de dos variedades de maíz (Zea mays L.) con fertilización mineral y orgánica en las condiciones de la Amazonía ecuatoriana. La investigación se desarrolló en el Centro de Investigación, Postgrado y Conservación Amazónica (CIPCA), en el cantón Arosemena Tola, provincia Napo para la variedad Tusilla y en la Granja Integral Ortiz ubicada en el cantón Sucúa, provincia de Morona Santiago para la variedad Zhubay. En ambos experimentos se utilizó un diseño en bloques completamente al azar con tres tratamientos (fertilización química, orgánica y testigo) y cuatro repeticiones. Se evaluaron los componentes del rendimiento y rendimiento agrícola. Se realizaron los análisis de variancia y prueba de media Tukey al 5\% para determinar la diferencia estadística entre las distintas variables. Se demuestra que las dos variedades locales muestran una buena producción por planta de 97, 144 y $94 \mathrm{~g} \mathrm{planta}^{-1}$ en la variedad Zhubay y de 94, 138 y 89 g planta $^{-1}$ en la variedad Tusilla según tratamientos, químico, orgánico y testigo respectivamente. Igualmente, el rendimiento en grano resulta muy favorable para variedades locales en estas condiciones con valores de 6.0; 9.0 y $5.8 \mathrm{Mg} \mathrm{ha}^{-1}$ en la variedad Zhubay y de $4.2 ; 5.7$ y $3.7 \mathrm{Mg}$ $\mathrm{ha}^{-1}$ en la variedad Tusilla. La variedad Zhubay obtuvo mejor comportamiento en todos los indicadores. En ambos casos cuando se aplica fertilizante orgánico se obtienen mejores resultados en las variables evaluadas, lo cual indica la posibilidad de utilizar variedades locales y fertilización orgánica en la producción de maíz en la región sin dependencia de insumos externos.

Palabras clave: Rendimiento, maíz, fertilización, paisaje amazónico.
Rec.: 25.05.2019. Acept.: 24.01.2020 Publicado el 30 de junio de 2020 Abstract

$\mathrm{T}$ he maize is one of the main grains in Ecuador, of great importance for indigenous peoples, who consider it a generator of life. In the Amazon region, the Tusilla and Zhubay varieties have been cultivated for many years, however, there are no agronomic studies to determine a production model for the region. Based on this, the objective of this work was to evaluate the productive development of two varieties of corn (Zea mays L.) with mineral and organic fertilization under the conditions of the Ecuadorian Amazon. The research was carried out in the Amazon Research, Postgraduate and Conservation Center (CIPCA), in the Arosemena Tola canton, Napo province for the Tusilla variety and in the Ortiz Integral Farm located in the Sucúa canton, Morona Santiago province for the Zhubay variety. In both experiments a completely randomized block design was used with three treatments (chemical, organic and control fertilization) and four repetitions. Agricultural yield and yield components were evaluated. Variance analysis and 5\% Tukey mean test were performed to determine the statistical difference between the different variables. It is shown that the two local varieties show a good production per plant of 97, 144 and $94 \mathrm{~g} \mathrm{plant}^{-1}$ in the Zhubay variety and 94, 138 and 89 g plant $^{-1}$ in the Tusilla variety according to treatments, chemical, organic and control respectively. Likewise, the grain yield is very favorable for local varieties in these conditions with values of $6.0 ; 9.0$ and $5.8 \mathrm{Mg}$ ha-1 in the Zhubay variety and $4.2 ; 5.7$ and $3.7 \mathrm{Mg}$ $\mathrm{ha}^{-1}$ in the Tusilla variety. The Zhubay variety obtained better performance in all indicators. In both cases, when organic fertilizer is applied, better results are obtained in the evaluated variables, which indicates the possibility of using local varieties and organic fertilization in the production of corn in the region without dependence on external inputs.

Keywords: Yield, corn, fertilization, Amazonian landscape. 


\section{Introducción}

$\mathrm{E}$ 1 maíz (Zea mays L.), originario de Centro América y zonas altas Andinas, es el cultivo que presenta mayor diversidad en la textura del grano, especialmente en sus formas nativas (Torres et al., 2012) lo que permite tener un sin número de variedades por localidades. Ocupa una posición destacada en la agricultura de América Latina, al destinarse tanto para consumo humano como animal. Hoy en día es uno de los cereales más cultivados en todo el mundo (Hernández y Soto, 2012).

En el Ecuador el maíz es un cultivo de mucha importancia económica, se siembra en la costa, sierra y la Amazonía bajo diferentes condiciones ambientales de temperatura, humedad, régimen de lluvias, luminosidad y suelos. Para ello, se siembran distintas variedades de maíz según las zonas, en la serranía ecuatoriana, se siembran variedades como: el chaucho, huandango, mishca, chillos, blanco blandito, cuzco ecuatoriano y shima, mientras que en la Amazonía las variedades Tusilla y Zhubay; y en la Costa Ecuatoriana se siembran granos de maíz amarillo duro y blancos duros. (Chaqui, 2013).

Las grandes empresas que multiplican semillas generan su propia tecnología de producción, la cual incluye el manejo agronómico, definiendo densidades de población y fertilización por la importancia que tienen estos factores en el rendimiento en granos. Por tanto, instituciones públicas y las universidades, también deben desarrollar tecnología de producción para las variedades mejoradas que generan y liberan comercialmente (Ortiz-Trejo et al., 2005 y TadeoRobledo et al., 2012). Esta información es útil para el productor, que, entre otros aspectos debe elegir el fertilizante y el método de aplicación que más se adapte a sus posibilidades, procurando apegarse a las recomendaciones regionales. Por ejemplo, en México se han reportado 59 razas de maíz criollo las cuales presentan diversas características agro-morfológicas que prácticamente le permiten al cultivo de maíz crecer en casi cualquier condición de clima y suelo (Ron Parra et al., 2006).

El manejo de la fertilización representa un factor importante en el proceso de producción por la influencia tan marcada que tiene sobre el rendimiento y sus componentes, lo cual ha sido comprobado en diferentes cultivos en condiciones amazónicas (Alemán et al., 2018). Bajo este enfoque, se ha señalado que para la obtención de rendimientos adecuados y sostenidos en el tiempo se hace necesario integrar la fertilidad y el manejo del cultivo (Bravo et al., 2018). Esto especialmente cobra mayor relevancia en los paisajes amazónicos donde los suelos se caracterizan por presentar $\mathrm{pH}$ ácidos, presencia de aluminio intercambiable, baja disponibilidad de nutrientes en especial fósforo $(\mathrm{P})$ disponible y de base intercambiables $\left(\mathrm{K}^{+} ; \mathrm{Ca}^{2+} \mathrm{y} \mathrm{Mg}^{2+}\right)$ ((Bravo et al., 2017). En términos prácticos significa que cualquier cambio de uso del suelo hacia cultivos agrícolas hace necesario la aplicación de fertilizantes ya sea en forma orgánica e inorgánica que suplan sus requerimientos (Bravo et al., 2018). Álvarez, Mendoza, Panizo y Sevillano (2017), en un experimento en el Instituto Nacional de Innovación Agraria-INIA (La Molina, Lima, Perú) donde determinaron el efecto de compost maduro y fertilización con urea sobre el rendimiento de maíz amarillo duro, obtuvieron rendimientos estadísticamente similares en los dos experimentos.

Bajo esta perspectiva, el objetivo de este trabajo consistió en evaluar el desarrollo productivo de dos variedades locales de maíz (Zea mays L.) con fertilización mineral y orgánica en el paisaje agrícola de la Amazonía Ecuatoriana

\section{Materiales y métodos}

\section{Localización de los experimentos}

El experimento con la variedad local Zhubay se desarrolló en la Granja Integral Ortiz ubicada en la parroquia Santa Marianita de Jesús, del cantón Sucúa, Provincia de Morona Santiago.

La parroquia de Santa Marianita de Jesús tiene una superficie de $58.35 \mathrm{Km}^{2}$ que representa el $4.54 \%$ del total de la superficie del cantón Sucúa, se encuentra ubicado en la parte Central Norte del cantón, geográficamente entre los puntos; $2^{\circ} 20^{\prime} 23^{\prime \prime} \mathrm{S}, 78^{\circ}$ $14^{\prime} 36^{\prime \prime} \mathrm{W}$, hasta $2^{\circ} 26^{\prime} 12^{\prime \prime} \mathrm{S}, 78^{\circ} 07^{\prime} 23^{\prime \prime} \mathrm{W}$, y sus límites son: al Norte: Cantón Morona, Al Sur, este y oeste: Cabecera cantonal Sucúa.

La temperatura media anual es de $21.8{ }^{\circ} \mathrm{C}$, y la altitud de $850 \mathrm{msnm}$. La precipitación media anual de $2000 \mathrm{~mm}$ con meses de lluvia que van de febrero a julio, con una transición para el cambio en el mes de agosto y los meses menos lluvioso corresponden de septiembre a enero.

Los suelos se relacionan directamente con la generación de movimientos en masa. En el cantón Sucúa se tiene suelos inceptisoles y entisoles, los cuales están presentes en la mayor parte del territorio. Con una capa arable que va desde $15 \mathrm{~cm}$ a $100 \mathrm{~cm}$ de profundidad (Espinosa, Moreno, Bernal, 2018). Están constituidos por depósitos cuaternarios volcánicos fluviales, que se asientan sobre formaciones de los períodos jurásico y cretáceo.

El uso de estos suelos es muy diverso y variado, en las áreas de pendientes son más apropiadas para la reforestación mientras que los suelos de depresiones 

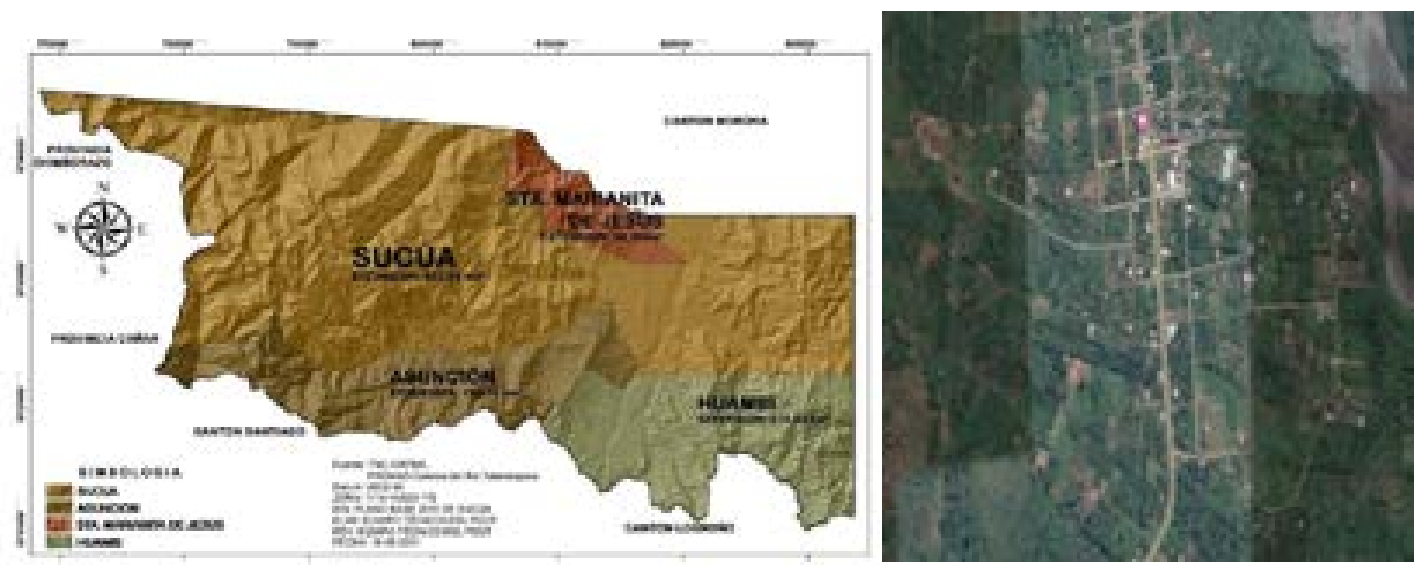

Figura 1. Localización del área experimental Granja Integral Ortiz, cantón Sucúa, provincia Morona Santiago

con drenaje artificial pueden ser cultivados porque son suelos fértiles.

\section{Tratamientos}

Considerando los resultados del análisis de suelo donde se encontraron contenidos altos de macroelementos y un pH ligeramente ácido, se consideró la aplicación de urea en el tratamiento químico, adicional una aplicación de urea vía foliar buscando una mayor asimilación de este nutriente por la planta.

1. Para el tratamiento con fertilizante químico se utilizó urea $222 \mathrm{~kg} \mathrm{ha}^{-1}$ el $50 \%$ (1.8 $\mathrm{g} \mathrm{planta}^{-1}$ ) al momento de la siembra y a los 21 días de la germinación el otro $50 \%\left(1.8 \mathrm{~g} \mathrm{planta}^{-1}\right) \mathrm{y}$ urea disuelta en agua a los 60 días mediante aplicación foliar en dosis de $1 \mathrm{~g} \mathrm{~L}^{-1}$ de agua.

2. Para el tratamiento orgánico se utilizó ecoabonaza a razón de $3416 \mathrm{~kg} \mathrm{ha}^{-1}$ al momento de la siembra (54.66 $\left.\mathrm{g} \mathrm{planta}^{-1}\right)$ y mediante aplicación foliar a los 21 y 60 días biol $1 \mathrm{~L} 5 \mathrm{~L}^{-1}$ de agua.

El experimento con la variedad local Tusilla se efectuó en el "Centro de Investigación, Posgrado y Conservación Amazónica (CIPCA), que se encuentra situado en la Región Amazónica Ecuatoriana, en la Provincia de Pastaza y Napo, en el Cantón Santa Clara y Carlos Julio Arosemena Tola; vía Napo Km. 44 con una altitud de 443 a $1137 \mathrm{msnm}$. La temperatura promedio es de $24^{\circ} \mathrm{C}$, con clima tropical húmedo y precipitación anual entre 3654.5 y $5516 \mathrm{~mm}$. El CIPCA comprende 2840,28 ha, con un $70 \%$ de bosque primario, con vegetación caracterizada por bosques húmedos lluviosos tropicales; este escenario amazónico cuenta con una alta diversidad florística y faunística" (Ramírez, González, Andrade, \& Torres, 2016).

1. Para el tratamiento con fertilizante químico en la siembra se aplicó la fórmula NPK (8-20-20) a razón de $30 \mathrm{~g} \mathrm{planta}^{-1}$ y a los 30 días de la geminación se aplicó Urea (N-46) a razón de $35 \mathrm{~g} \mathrm{~m}^{-2}$.

2. Para el tratamiento orgánico se utilizó compost, obtenido del Programa de Abonos del CIPCA, a razón de $5 \mathrm{~kg} \mathrm{~m}^{-2}$.

\section{Evaluaciones realizadas en ambos experimentos}

En el momento de la cosecha se seleccionaron 5 plantas que se encontraban en competencia intraespecífica perfecta para evaluar los componentes del rendimiento tales como: a) Número de hileras por mazorca, b) Número de granos por hilera, c) Largo de la mazorca, d) Diámetro de la mazorca, e) Diámetro de la tusa, f) Peso de 100 granos, g) Rendimiento por planta (gramos), h) rendimiento agrícola $\left(\mathrm{Mg} \mathrm{ha}^{-1}\right)$

\section{Análisis estadístico de los resultados}

Se utilizó el modelo estadístico correspondiente al diseño experimental y se realizó los ANOVA con el programa estadístico SPSS versión 21 y la prueba de rango múltiple de Tukey al 5\% para las variables que mostraron diferencias significativas.

\section{Resultados y discusión}

\section{Características de los suelos en las áreas de estudio}

Las características químicas de los suelos asociados a las áreas bajo estudio se muestran en el cuadro 1. Se encontró que el proceso de acidificación, la acumulación de materia orgánica y la disponibilidad de nutrientes fueron diferentes de acuerdo a la zona de estudio.

El nivel de acidez del suelo osciló de ligeramente ácido (Sucúa) a ácido (CIPCA), lo cual puede influir en distintos procesos biológicos y en especial sobre la disponibilidad de nutrientes (McGrath et al., 2014). La acidificación en regiones tropicales húmedas como la Amazonía Ecuatoriana, con un clima caracterizado por 


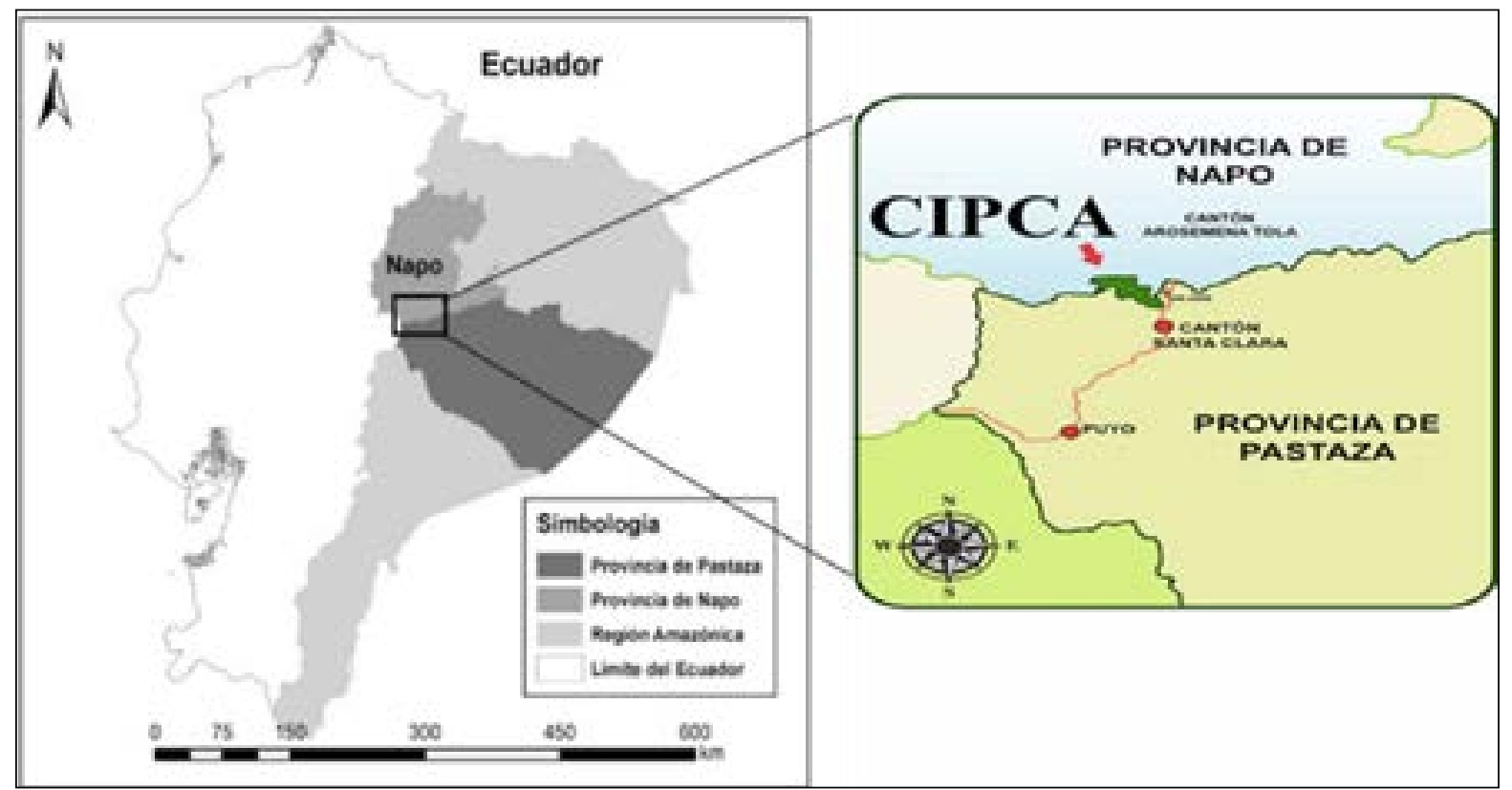

Figura 2. Ubicación geográfica del CIPCA

Cuadro 1. Características químicas de los suelos en las áreas de estudio

\begin{tabular}{|c|c|c|}
\hline Variables & Sucúa & CIPCA \\
\hline $\mathrm{pH}$ & 6.20 LAc & $5.22 \mathrm{Ac}$ \\
\hline Materia orgánica (\%) & $5.75 \mathrm{~A}$ & $3.5 \mathrm{M}$ \\
\hline Amonio $\left(\mathrm{NH}_{4}^{+}\right) \cdot \mathrm{mg} \mathrm{kg}^{-1}$ & $67.40 \mathrm{~A}$ & $27.46 \mathrm{~B}$ \\
\hline Fósforo $(\mathrm{P}) . \mathrm{mg} \mathrm{kg}^{-1}$ & $42.70 \mathrm{~A}$ & $4.86 \mathrm{~B}$ \\
\hline Potasio $\left(\mathrm{K}^{+1}\right)$. meq 100 & $0.91 \mathrm{~A}$ & $0.10 \mathrm{~B}$ \\
\hline Calcio $\left(\mathrm{Ca}^{+2}\right)$. meq $100 \mathrm{~g} \mathrm{~s}^{-1}$ & $15.80 \mathrm{~A}$ & $2.00 \mathrm{~B}$ \\
\hline Magnesio $\left(\mathrm{Mg}^{+2}\right)$. meq $100 \mathrm{ml}$ & $2.59 \mathrm{~A}$ & $0.48 \mathrm{~B}$ \\
\hline Cobre $(\mathrm{Cu}) \mathrm{mg} \mathrm{kg}^{-1}$ & $5.93 \mathrm{~A}$ & $8.60 \mathrm{~A}$ \\
\hline Hierro $(\mathrm{Fe}) . \mathrm{mg} \mathrm{kg}^{-1}$ & $148.70 \mathrm{~A}$ & $200.85 \mathrm{~A}$ \\
\hline Azufre (S). $\mathrm{mg} \mathrm{kg}^{-1}$ & $5.50 \mathrm{~B}$ & $13.19 \mathrm{M}$ \\
\hline Zinc $(\mathrm{Zn}) . \mathrm{mg} \mathrm{kg}^{-1}$ & $12.00 \mathrm{~A}$ & $1.68 \mathrm{~B}$ \\
\hline Boro (B) $\mathrm{mg} \mathrm{kg}^{-1}$ & $0.24 \mathrm{~B}$ & $0.45 \mathrm{~B}$ \\
\hline
\end{tabular}

Lac: Ligeramente ácido; Ac: Acido; A. Alto, M. Medio; B: Bajo

altas precipitaciones ejerce una influencia primordial sobre la edafogénesis que favorece la lixiviación de las bases $\left(\mathrm{Ca}^{2+}, \mathrm{Mg}^{2+}, \mathrm{K}^{+}\right)$, lo que induce a un predominio de minerales poco alterables y de arcillas simples como el cuarzo, caolinita, gibsita y óxidos de hierro, confiriéndoles ciertas características morfológicas y el descenso de los parámetros asociados a la fertilidad principalmente el pH (Custode y Sourdat, 1986; Gardi et al. 2014).

El nivel de materia orgánica fue mayor en la localidad de Sucúa con respecto al suelo del CIPCA, categorizados como alto y medio respectivamente. Estos altos reservorios de carbono están asociados a los antecedentes de uso con bosque, característicos de la región amazónica (Nieto y Caicedo, 2012). Algunos autores señalan que los reservorios de carbono se corresponden a los procesos de formación de ácidos húmicos, con sus beneficios derivados en forma de mejora de la estabilidad estructural, de la retención de agua, la disponibilidad de nutrientes y actividad microbiana (Martínez et al., 2008; Gallardo, 2016).

La concentración de nutrientes mostró un patrón similar al contenido de materia orgánica, con valores categorizados como alto en la mayoría de los casos en Sucúa, en comparación con el suelo del CIPCA cuyos valores fueron calificados como bajos (cuadro 1). Los resultados del suelo del CIPCA coinciden con trabajos previos en distintos paisajes de la amazonia y bajo diferentes usos de la tierra que ponen en evidencia los bajos contenidos de nutrientes en condiciones amazónicas, lo cual es un factor limitante en la productividad del ecosistema (Bravo et al., 2017) y confirman que el cambio de uso hacia sistemas agrícolas requiere de la aplicación de fertilizantes para satisfacer los requerimientos nutricionales del cultivo.

Influencia de la fertilización química y orgánica sobre los componentes del rendimiento de la planta en las variedades locales Zhubay y Tusilla

La longitud y diámetro de la mazorca, el diámetro de la tusa y el número de hileras de granos de la mazorca no difieren estadísticamente entre los tratamientos en ninguna de las variedades, (cuadros 2 y 3 ), resultando ligeramente superior en la variedad 
Cuadro 2. Influencia de la fertilización química y orgánica sobre los componentes del rendimiento variedad local Zhubay en Sucúa, Morona Santiago

\begin{tabular}{llllrrr}
\hline \multicolumn{1}{c}{ Tratamientos } & \multicolumn{1}{c}{$\mathbf{N}^{\mathbf{0}} \mathbf{H m}$} & $\mathbf{N}^{\circ} \mathbf{g h}$ & $\mathbf{D m}$ & $\mathbf{D t}$ & $\mathbf{L m}$ & P100g \\
\hline Químico & $12.13 \mathrm{a}$ & $32.93 \mathrm{~b}$ & $3.33 \mathrm{a}$ & $1.62 \mathrm{a}$ & $17.22 \mathrm{a}$ & $25.21 \mathrm{~b}$ \\
Orgánico & $12.25 \mathrm{a}$ & $43.62 \mathrm{a}$ & $3.46 \mathrm{a}$ & $1.60 \mathrm{a}$ & $15.69 \mathrm{a}$ & $35.29 \mathrm{a}$ \\
Testigo & $11.75 \mathrm{a}$ & $28.44 \mathrm{~b}$ & $3.15 \mathrm{a}$ & $1.56 \mathrm{a}$ & $16.59 \mathrm{a}$ & $22.48 \mathrm{c}$ \\
\hline Valor F & 0.163 & 36.06 & 1.135 & 1.002 & 4.135 & 51.52 \\
Nivel de significancia & 0.850 & 0.000 & 0.000 & 0.000 & 0.000 & 0.000 \\
\hline
\end{tabular}

Nota: $\mathrm{N}^{0} \mathrm{Hm}=$ número de hileras por mazorca; $\mathrm{N}^{\mathbf{0}} \mathrm{gh}=$ número de granos por hilera; $\mathrm{Dt}=$ diámetro de la tusa $(\mathrm{cm}) ; \mathrm{Dm}=$ diámetro de la mazorca $(\mathrm{cm}) ; \mathrm{Lm}=$ largo de la mazorca $(\mathrm{cm}) ; \mathrm{P} 100 \mathrm{~g}=$ peso de 100 granos (g). Tukey $\mathbf{p}<0.05$. Letras distintas para la misma columna indican diferencias significativas entre tratamientos.

Cuadro 3. Influencia de la fertilización química y orgánica sobre los componentes del rendimiento variedad Tusilla en Arosemena Tola, Napo

\begin{tabular}{ccccccc}
\hline Tratamientos & $\mathbf{N}^{\mathbf{0}} \mathbf{H m}$ & $\mathbf{N}^{\mathbf{0}} \mathbf{g h}$ & $\mathbf{D m}$ & $\mathbf{D t}$ & $\mathbf{L m}$ & $\mathbf{P 1 0 0 g}$ \\
\hline Químico & $11.43 \mathrm{a}$ & $29.93 \mathrm{~b}$ & $3.71 \mathrm{a}$ & $1.60 \mathrm{a}$ & $16.52 \mathrm{a}$ & $25.20 \mathrm{~b}$ \\
Orgánico & $12.78 \mathrm{a}$ & $40.32 \mathrm{a}$ & $3.43 \mathrm{a}$ & $1.57 \mathrm{a}$ & $15.78 \mathrm{a}$ & $30.08 \mathrm{a}$ \\
Testigo & $10.25 \mathrm{a}$ & $26.04 \mathrm{~b}$ & $3.40 \mathrm{a}$ & $1.50 \mathrm{a}$ & $14.87 \mathrm{a}$ & $18.32 \mathrm{c}$ \\
\hline Valor F & 0.153 & 33.04 & 1.121 & 1.001 & 4.521 & 48.52 \\
Nivel de significancia & 0.750 & 0.000 & 0.000 & 0.000 & 0.000 & 0.000 \\
\hline
\end{tabular}

Nota: $\mathrm{N}^{\circ} \mathrm{Hm}=$ número de hileras por mazorca; $\mathrm{N}^{0} \mathrm{gh}=$ número de granos por hilera; $\mathrm{Dt}=$ diámetro de la tusa $(\mathrm{cm}) ; \mathrm{Dm}=$ diámetro de la mazorca $(\mathrm{cm}) ; \mathrm{Lm}=$ largo de la mazorca $(\mathrm{cm}) ; P 100 \mathrm{~g}=$ peso de 100 granos $(\mathrm{g})$. Tukey $\mathbf{p}<\mathbf{0 . 0 5}$. Letras distintas para la misma columna indican diferencias significativas entre tratamientos.

Zhubay, excepto el diámetro de la tusa. Esto indica que los nutrientes que estuvieron a disposición por las plantas fueron suficientes para la formación de estos parámetros productivos, sin embargo, los valores obtenidos son inferiores a los reportados por Darquea, Alemán y Domínguez (2018), quienes reportan valores de 16 hileras por mazorca en una variedad local de maíz a igual densidad de población.

El número de granos por hilera fue mayor cuando se aplicó fertilizante orgánico con diferencia estadística para los otros dos tratamientos que no difieren entre sí para este indicador en ninguna de las dos variedades. En todos los casos en la variedad Zhubay se muestran los mejores valores con 43, 32 y $28 \mathrm{~g}_{\text {hilera }}{ }^{-1}$ para el fertilizante orgánico, químico y testigo respectivamente respecto a la variedad Tusilla con valores de 40, 29 y 26 g hilera $^{-1}$.

Estos resultados son superiores a los encontrados por Darquea, Alemán y Domínguez (2018) quienes reportan valores de 25 granos hilera $^{-1}$. Con el fertilizante orgánico se obtuvo un mayor P100g en las dos variedades con diferencia estadística para el fertilizante químico y éste a su vez para el testigo, e igualmente superior en la variedad Zhubay.

El aprovechamiento de los fertilizantes orgánicos cobra cada día mayor importancia como medio eficiente de reciclaje racional de nutrientes que ayuda al crecimiento de las plantas y devuelven al suelo muchos de los elementos extraídos durante el proceso productivo (Cerrato, Leblanc y Kameko, 2007). Díaz et al. (2009) reporta un peso de semillas por mazorca de $136.5 \mathrm{~g}$. Los campesinos generalmente no emplean el P100g para seleccionar sus accesiones, pero al hacer la selección basada en alguno de estos otros caracteres, están indirectamente influyendo en el P100g.

Influencia de la fertilización química y orgánica sobre el rendimiento por planta y rendimiento agrícola en las variedades locales Zhubay y Tusilla

En cuanto al rendimiento en grano expresado en g planta ${ }^{-1}$ o $\mathrm{Mg} \mathrm{ha}^{-1}$ (Figuras 3 y 4) el tratamiento con fertilizante orgánico presentó diferencia significativa para el tratamiento químico y el testigo con valores muy buenos para estas condiciones edafoclimáticas superiores a los 5.7 y $9.0 \mathrm{Mg} \mathrm{ha}^{-1}$ para las variedades Tusilla y Zhubay respectivamente.

Según García et al. (2013), la fertilización orgánica benefició el desarrollo del maíz y las diferencias detectadas en los tratamientos evaluados se relacionaron con el contenido de elementos nutritivos en la planta y sus comunidades microbianas. Estos resultados resultan superiores a los obtenidos por Darquea, Alemán y 


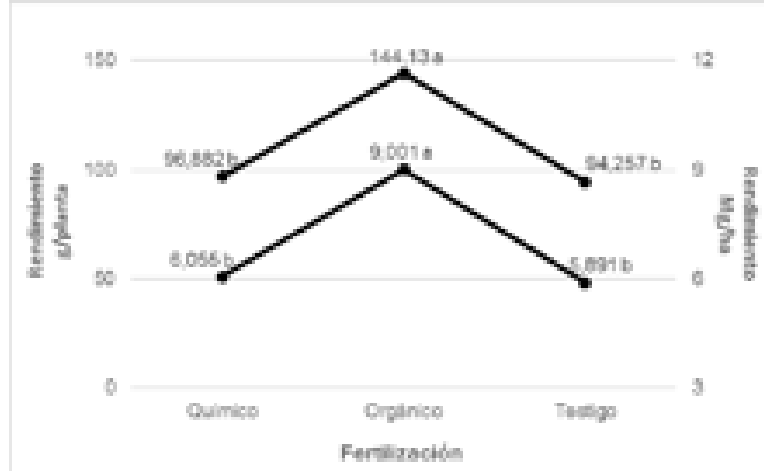

Figura 3. Influencia de la fertilización química y orgánica sobre los rendimientos por planta y agrícola de la variedad local Zhubay en Sucúa, Morona Santiago. Tukey $p<0.05$. Letras distintas para la misma columna indican diferencias significativas entre tratamientos

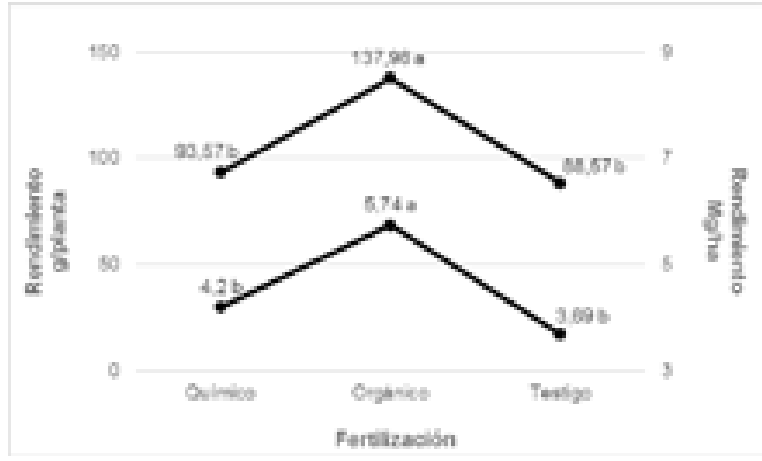

Figura 4. Influencia de la fertilización química y orgánica sobre los rendimientos por planta y agrícola de la variedad local Tusilla en Arosemena Tola, Napo. Tukey $\mathbf{p}<\mathbf{0 , 0 5}$. Letras distintas para la misma columna indican diferencias significativas entre tratamientos

Domínguez (2018) con rangos entre 5 y $6 \mathrm{Mg}$ ha $^{-1}$ en una variedad local en las condiciones del CIPCA en el Cantón Arosemena Tola. Con el abono orgánico se produce entre 40 y $50 \mathrm{~g}$ más de rendimiento por planta. Estos resultados difieren a los conseguidos por Yánez et al. (2010), en experimentos en dos localidades de la Provincia de Bolívar (Ecuador), aplicando la metodología del elemento faltante, donde no encontraron diferencias significativas para la producción de grano. Tampoco encontraron diferencias entre tratamientos Álvarez et al. (2017) en un estudio sobre el efecto de fuentes orgánicas y sintéticas de nitrógeno en un cultivo de maíz amarillo duro en La Molina, Perú.

López, Díaz, Martínez y Valdez (2001) y Forero, Serrano y Almanza (2014) obtuvieron resultados diferentes a los de este estudio con mayores rendimientos al aplicar fertilizante químico comparado con el tratamiento con abono orgánico. Los resultados de estos autores, diferentes al conseguido en este trabajo, podrían deberse a las condiciones edafoclimáticas donde se realizaron los experimentos, para lo cual debemos considerar que las altas precipitaciones en la Amazonía hacen que se lave fácilmente el fertilizante químico lo que no sucede con el abono orgánico que a más de mantener los elementos químicos necesarios mejora la estructura del suelo. Esto coincide con Flores et al. (2004), cuando plantean que la urea constituye uno de los fertilizantes nitrogenados más empleados en la agricultura, a pesar de tener dos inconvenientes importantes: su gran solubilidad en agua y la posibilidad de sublimar, que provocan grandes pérdidas del fertilizante. También García et al. (2013) obtienen un rendimiento aceptable de grano con aplicación de vermicomposta como fertilizante orgánico, concluyendo que la aplicación de los abonos orgánicos vermicomposta y supermagro mostró que un buen rendimiento de maíz criollo de Sinaloa es factible. Resultado similar obtienen Álvarez et al. con fertilización nitrogenada.

Varias investigaciones han mostrado aumento en la disponibilidad de nutrientes usando estiércoles compostados como gallinaza, lombricompost, Galindo (2012), porquinaza Xiang et al. (2012). Otros autores se refieren a que los abonos orgánicos pueden brindar beneficios al adicionarlos a suelos de bajo pH, Gómez y Tovar (2008).

\section{Conclusiones}

T a fertilización orgánica benefició el desarrollo del maíz, los componentes del rendimiento y el rendimiento agrícola en ambas localidades y variedades evaluadas, lo cual indica la posibilidad de utilizar variedades locales y fertilización orgánica en la región sin dependencia de insumos externos. En base a ello, se sugiere que las variedades locales Zhubay y Tusilla deben ser consideradas en los sistemas de producción para las zonas estudiadas dado su buen comportamiento en los indicadores productivos en especial con la fertilización orgánica.

\section{Bibliografía}

Alemán-Pérez, R; Bravo Medina, C.; Fargas-Clua, M. 2018. Fertilización orgánica en cultivos de lechiga (Lactuca sativaL) y rábano (Rphanus sativu L) en la Amazonía Ecuatoriana. Edición Associació Catalan d Enginyeria Sense Fronteres. Puyo, Ecuador. 96 pp.

Álvarez, R. S., Chuquija, J. C., Mendoza, C. C., Panizo, R. S., \& Sevillano, R. B. (2017). Fuentes y dosis de nitrógeno en la productividad del maíz amarillo duro bajo dos sistemas de siembra. In Anales Cientificos (Vol. 78, No. 2, pp. 232-240). Universidad Nacional Agraria La Molina. 
Bravo Medina, C.; Alemán-Pérez, R., Días, L., Fargas-Clua, My Guell-Ordis,J. 2018. El recurso suelo y su fertilidad integral. En: Alemán- Pérez, R; Bravo Medina, C.; Fargas-Clua, M. 2018. Fertilización orgánica en cultivos de lechiga (Lactuca sativaL) y rábano (Rphanus sativu L) en la Amazonía Ecuatoriana. Edición Associació Catalan d Enginyeria Sense Fronteres. Puyo, Ecuador. 96 pp.

Bravo, C; Ramírez, A.; Marín, H.; Torres, B.; Alemán, R.; Torres, R.; Navarrete, H. y Changoluisa, D. 2017. Factores asociados a la fertilidad del suelo en diferentes usos de la tierra de la Región Amazónica Ecuatoriana. Rev. Electrón. vet. Volumen $18 \mathrm{~N}^{\mathrm{o}}$ 11: 1-17.

Chaqui, C. 2013. Formación de una variedad experimental de maíz amarillo suave (Zea mays l.) tipo "mishca" a partir de medios hermanos y hermanos completos. Tumbaco, Pichincha. UCE.

Cerrato, M. E., Leblanc, H. A., \& Kameko, C. (2007). Potencial de mineralización de nitrógeno de Bokashi, compost y lombricompost producidos en la Universidad Earth. Tierra Tropical, 3(2), 183-197.

Custode. E y Sourdat M. 1986. Paisajes y suelos de la Amazonía ecuatoriana: entre la conservación y la explotación. Revista del Banco Central del Ecuador 24: 325-339.

Darquea W., Alemán R. y Dominguez J. 2018. Respuesta de un genotipo local de maíz (Zea Mays L.) de la Amazonía ecuatoriana a diferentes densidades de población. Memorias del Primer Congreso Internacional Alternativas Tecnológicas para la producción agropecuaria sostenible en la Amazonía Ecuatoriana. 151-153.ISBN 978-994235-604-8.

Díaz, G., Sabando, F., Zambrano, S., Vásconez, G. 2009. Evaluación productiva y calidad del grano de cinco híbridos de maíz (Zea mays 1.) en dos localidades de la provincia de los Ríos. Ciencia y Tecnología. 2(1) 15-23.

Espinosa, J., Moreno, J., Bernal, G. 2018. The soils of Ecuador. The World Soils Book Series. ISBN 978-3319-25317-6. https://doi.org/10.1007/9783-319-25319-0_1

Flores, D., García, T., Martínez, R., Martínez, A., López, A., \& Ruiz, E. (2004). Síntesis y aplicación del producto de condensación de la urea con el furfural en el cultivo de maíz. Cultivos Tropicales, 25(1).

Forero, U., Serrano, C., \& Almanza, M. (2014). Effect of organic amendments and chemical fertilizer application in maize production. Cultura Cientifica, (12), 46-55.

Galindo, W.R. 2012. Efecto de dos dosis de abonos orgánicos en el desarrollo y un componente del rendimiento del cultivo de la soya (Glycine max (L.) Merril), en áreas de la Universidad de Granma, 37pp., Tesis de grado, Unidad Académica de Ciencias Agropecuarias y Recursos Naturales, Universidad Técnica de Cotopaxi, Colombia.

Gallardo, J. F. 2016. La materia orgánica del suelo: Residuos orgánicos, humus, compostaje y captura de carbono.
Editorial SiFyQA, Salamanca. ISBN: 978-84-9374377-2. $392 \mathrm{pp}$

García, J. A., Villarreal, M., Sánchez, P., Parra, S., Hernández, S. (2013): Fertilización con vermicomposta en maíz criollo y su tasa de descomposición en el suelo. Revista de Investigación Agraria y Ambiental - Volumen 4 Número 1 - enero-junio de 2013 - ISSN 2145-6097.

Gardi, C., Angelini, M., Barceló, S., Comerma, J., Cruz Gaistardo, C., Encina Rojas, A., Jones, A., Krasilnikov, P., Mendonça Santos Brefin, M. \& Montanarella, L. 2014. Atlas de suelos de America Latina y el Caribe, Luxembourg: Comisión Europea, Oficina de Publicaciones de la Unión Europea.

Gómez, A. y Tovar, X. 2008. Elaboración de un abono orgánico fermentado a partir de residuos de flores (pétalos de rosa) y su caracterización para uso en la producción de albahaca (Ocimum basilicum L.), 104pp., Pontificia Universidad Javeriana, Facultad de Ciencias, Bogotá, Colombia.

Hernández Córdova, N., y Soto Carreño, F. 2012. Influencia de tres fechas de siembra sobre el crecimiento y rendimiento de especies de cereales cultivadas en condiciones tropicales. Parte I. cultivo del maíz (Zea mays L.). Cultivos Tropicales, 33 (2), 44-49

López Mtz, J. D., Díaz Estrada, A., Martínez Rubin, E., \& Valdez Cepeda, R. D. (2001). Abonos orgánicos y su efecto en propiedades físicas y químicas del suelo y rendimiento en maíz. Terra latinoamericana, 19(4).

Martínez, E; Fuentes JP y Acevedo-H, E. 2008. Carbono orgánico y propiedades del suelo. J. Soil Sc. Plant Nutr. 8 (1) (68-96).

McGrath, J. M., Spargo, J. y Penn J. 2014. Soil Fertility and Plant Nutrition. Encyclopedia of Agriculture and Food Systems. 5: 165-184.

Nieto, C. \& Caicedo, V. 2012. Análisis reflexivo sobre el desarrollo agropecuario sostenible en la Amazonía Ecuatoriana. INIAP-EECA. Publicación Miscelánea No 405, Joya de las Sachas, Ecuador. 102 p.

Ortiz-Trejo C., A. Espinosa-Calderón, H. S. Azpíroz-Rivero y S. Sahagún- Castellanos. 2005. Producción y tecnología de semillas de maíz del INIFAP para los Valles Altos y zonas de transición. INIFAP. CIRCE. Campo Experimental Valle de Toluca. Libro Técnico Número 3, Zinacantepec, estado de México.

Ramírez, A., González, J., Andrade, V., \& Torres, V. (2016). Efecto de los tiempos de conservación a temperatura ambiente, en la calidad del huevo de gallinas camperas (Gallus domesticus) en la Amazonia Ecuatoriana. REDVET. Revista Electrónica de Veterinaria, 17(12).

Ron, P.; Sánchez, J.; Jiménez, A.; Carrera, J.; Martín, J.; Morales, M.; De la Cruz, L.; Hurtado, S.; Mena, S. \& Rodríguez, J. (2006). Maíces Nativos del Occidente de México. I. Colectas 2004. Scientia-CUCBA 8(1): 1-139. ISBN: 970-27-0955-5. Editorial Tecnología y 
Aplicaciones Gráficas.

Tadeo-Robledo, M., Espinosa-Calderon, A., Chimal, N., Arteaga-Escamilla, I., Trejo- $\quad$ Pastor, V., CanalesIslas, E., Sierra-Macías, M. Valdivia-Bernal, R., GómezMontiel, N., Palafox-Caballero, A., Zamudio-González, B. 2012. Densidad de población y fertilización en híbridos de maíz androestériles y fértiles. Terra Latinoamericana, 30 (2), 157-164.

Torres, M., Bravo, A., Caviedes, M. y Arahana, V. 2012. Molecular and morphological characterization of S2 lines of black corn (Zea mays L.) from Ecuadorian Andes. Avances en Ciencias e Ingenierías. Publicado el

Xiang, L. Caixia, D., Yiren, L. Yanxia, L. Qirong, S. y Yangchun, X. 2012. Interactive effects from combining inorganic and organic fertilisers on phosphorus availability, Soil Research, 50, 607-615.

Yánez D., Valverde F. y Cartagena Y. 2010. Evaluación del elemento faltante en el cultivo de maíz (Zea mays L.) en la Provincia Bolívar. XII Congreso Ecuatoriano de la Ciencia del Suelo.17-19 noviembre de 2010. Santo Domingo- Ecuador. 